\title{
Detection of the MJO Signal from QuikSCAT
}

\author{
Anthony Arguez,* Mark A. Bourassa, and James J. O’Brien \\ Center for Ocean-Atmospheric Prediction Studies, Tallahassee, Florida
}

(Manuscript received 3 February 2005, in final form 20 May 2005)

\begin{abstract}
Wind data from the SeaWinds instrument on NASA's Quick Scatterometer (QuikSCAT) satellite are investigated to ascertain how well the surface manifestation of the Madden-Julian oscillation (MJO) can be resolved. The MJO signal is detected in nonfiltered gridded data using extended EOF analysis of the zonal wind field, overshadowed by annual, semiannual, and monsoon-related modes. After bandpass filtering with Lanczos weights, MJO signals are clearly detected in several kinematic quantities, including the zonal wind speed, the zonal pseudostress, and the velocity potential. Extraction of the MJO using QuikSCAT winds compares favorably with extraction using NCEP Reanalysis 2, except that the QuikSCAT signal appears to be more robust.

In addition, an alternative bandpass-filtering technique using variable filter weights near time series endpoints is presented. The method uses least squares minimization to match newly created frequency response functions in edge zones as closely as possible to the predetermined frequency response function of interior points. This method stands in contrast to the common practice of simply discarding those endpoints where a convolution cannot be computed.
\end{abstract}

\section{Introduction}

The Madden-Julian oscillation (MJO) is the primary mode of intraseasonal variability in the Tropics, although the signal is often more conspicuous in the Indian and western Pacific Oceans than it is over the eastern Pacific and the Atlantic. The oscillation exhibits a relatively broad frequency range, repeating anywhere from 20 to 100 days. However, much of the signal's spectral energy is concentrated in the 40-60-day band. First observed by the mode's namesakes in 1971, the MJO is somewhat unique among large-scale climatic signals in that its frequency range is its defining characteristic (Madden and Julian 1971). In fact, Madden and Julian originally dubbed this signal the 40-50 Day Oscillation. A secondary attribute of the MJO is its eastward, equatorially trapped propagation.

The MJO signal is fairly conspicuous in outgoing longwave radiation (OLR) data, which is a proxy for convective activity in the Tropics (Lau and Chan 1985).

\footnotetext{
* Current affiliation: National Climatic Data Center, Asheville, North Carolina.

Corresponding author address: Anthony Arguez, NCDC CAB Room 415, 151 Patton Avenue, Asheville, NC 28806.

E-mail: Anthony.Arguez@noaa.gov
}

MJO convective anomalies in the Indian and western Pacific Oceans account for a large portion of precipitation variability on intraseasonal time scales. However, the MJO signal is found in other variables: wind (Madden and Julian 1994), pressure (e.g., Kayano and Kousky 1998), and even sea surface temperature (Krishnamurti et al. 1988). Given the clear link between the MJO and tropical convection, the divergent component of the wind field plays a more visible role than the rotational component, although a growing body of evidence suggests that rotational effects cannot be discounted (e.g., Raymond 2001).

In the present study, the ability of the MJO to be identified in Quick Scatterometer (QuikSCAT) data is investigated. QuikSCAT is a relatively new tool that can be utilized to address outstanding MJO questions near the surface, such as the physics involved in the propagation and reinitiation mechanisms. First, it is necessary to assess the level to which QuikSCAT is able to capture the MJO signature. It will be shown that the MJO is indeed conspicuous in a prefiltered gridded QuikSCAT product, and that clear signals can be seen in the following variables after bandpass filtering over the MJO time scales: zonal wind, velocity potential, and zonal pseudostress at the surface. Moreover, we show that the average propagation speed over the Indian and western Pacific Oceans is on the order of $4 \mathrm{~m} \mathrm{~s}^{-1}$. The 
novelty of the present work is the ability to clearly detect the MJO in a gridded QuikSCAT dataset, as well as the presentation of a new bandpass-filtering algorithm that retains time series endpoints and can be utilized for real-time monitoring of the $\mathrm{MJO}$ and other climate phenomena. The QuikSCAT dataset can be utilized in the future to improve our understanding of MJO physics at the surface, a research avenue that has so far relied on reanalysis data.

Section 2 describes the QuikSCAT gridded product. The detection of the MJO in prefiltered QuikSCAT data is addressed in section 3. Section 4 highlights the results of analyses involving bandpass-filtered data. A summary and concluding remarks are in section 5 .

\section{QuikSCAT wind product}

\section{a. Scatterometer data}

Scatterometers are active microwave instruments that measure backscatter, primarily from capillary waves on the ocean's surface. Scatterometers are mounted on polar-orbiting satellites. Their motion relative to the earth's surface enables them to measure collocated backscatter from several orientations (azimuth directions and incident angles). Backscatter is a function of both wind speed and direction: given observations at enough orientations, wind speed and direction can be recovered (Wentz and Smith 1999). The observational accuracy is extremely good (Bourassa et al. 2003): $0.3 \mathrm{~m} \mathrm{~s}^{-1}$ and $3^{\circ}$ for the $\mathrm{Ku}-2000$ model function. These numbers represent one standard deviation in random errors. They are determined with in situ data that are closely collocated in time ( $<30 \mathrm{~s}$ difference), and the influence of differences in spatial collocation was considered by binning the collocated data as a function of difference in distance. These results are averaged across the swath, and in the absence of rain. The scatterometer backscatter observations that contribute to a single wind vector can be averaged over an approximately $70 \mathrm{~km} \times 70 \mathrm{~km}$ area; however, the spatial averaging is far from uniform. The wind vector observations were found to correspond marginally best with a 7-km length scale; the above estimates of uncertainty apply to this scale. More small-scale information is convolved into the scatterometer observations than was originally expected.

The influences of rain are functions of rain rate and wind speed (Weissman et al. 2002); however, all vectors suspected of being rain contaminated are flagged, regardless of the magnitude of the problem. For most storms there is little modification to many raincontaminated wind vectors: the wind signal dominates the influence of rain. However, in areas of low wind speed, the rain often leads to serious errors. For our scatterometer wind fields, all observations that are flagged as rain-contaminated are removed from consideration. Rather than instrument measurement error or rain contamination, the irregular temporal sampling (Schlax et al. 2001) at a given location by the scatterometer during its orbital cycle contributes the greatest errors during the mapping of wind data along the satellite observational swaths onto regular grids. For time series analysis, this problem is very severe for frequencies more rapid than several times the length of the orbital cycle, which is 4 days for QuikSCAT.

\section{b. The objective gridding technique}

The gridded scatterometer fields are produced using a variational approach adapted from Pegion et al. (2000). The key differences from Pegion et al. are the direct consideration of uncertainty and the construction of the background field. The constraints in the variational method are misfits to the observed vector components, a Laplacian penalty function, and a misfit to the curl of the background field. The constraint on the curl of the pseudostress couples the vector components and greatly reduces spurious curl-related ocean forcing. The weights for each of these constraints are determined through cross validation. The quality and sampling of the scatterometer observations are such that the objectively determined weights put most of the emphasis on the observation field.

\section{c. Experimental design}

From the zonal $\left(\tau^{x}\right)$ and meridional $\left(\tau^{y}\right)$ components of pseudostress, the zonal $(u)$ and meridional $(v)$ wind components are computed. From $u$ and $v$, the velocity potential $(\chi)$ is derived via a spectral method (see Krishnamurti et al. 1998). Only results of analyses of $u$, $\tau^{x}$, and $\chi$ are considered. The data span from $10^{\circ} \mathrm{N}$ to $10^{\circ} \mathrm{S}$ and from $40^{\circ} \mathrm{E}$ to $80^{\circ} \mathrm{W}$ on a $1^{\circ} \times 1^{\circ}$ grid, covering the deep Tropics of the Indian and Pacific Oceans. The data are temporally averaged into 392 consecutive pentads, from late July 1999 through early December 2004.

\section{Detection of the MJO from prefiltered QuikSCAT data}

\section{a. Methodology}

Obtaining a representative time series of the MJO is not a trivial task. The MJO is assumed to be present in the time series of every grid point in our domain. A variance decomposition technique is required to obtain a representative time series of the MJO for the entire domain. The need to consolidate geophysical data into 
the most energetic modes prompted the application of principal component analysis, or empirical orthogonal function (EOF) analysis, to geophysical space-time datasets. Kessler (2001) and Wheeler and Hendon (2004) are among the many works that have successfully utilized EOF analysis to define the MJO. A common problem with EOF analysis is that the spatial modes are stationary, an unattractive attribute when dealing with a propagating feature like the MJO. In addition, proper detection of the MJO with EOF analysis typically requires prior removal of low-frequency variability.

To overcome the shortcomings of EOF analysis on nonfiltered data, we utilize extended EOF analysis, which uses a covariance matrix extended by crosscovariances at different lags (e.g., see Weare and Nasstrom 1982). This allows the spatial pattern to move in time because propagating patterns (assuming the propagation speed is fairly uniform) will be spatially correlated at a given distance over a given time interval. Extended EOF analysis has been widely used to represent MJO variability (e.g., Lau and Chan 1985; Kayano and Kousky 1998; Myers and Waliser 2003). Consecutive plots of the lagged eigenfunctions clearly demonstrate propagating modes (a practical feature that EOF analysis lacks by its very nature). Moreover, since extended EOF analysis is able to differentiate between a propagating intraseasonal pattern (the MJO) and similar spatial signatures that are associated with lowfrequency variability, extended EOF analysis is preferable over EOF analysis for nonfiltered data in the MJO domain.

The justification for extracting the MJO time scale from space-time data is often misconstrued by some in the scientific community. A frequent concern is that this act constitutes statistical voodoo, observing a 4060 -day oscillation only after bandpass filtering over the 40-60-day time scale. Based on the superposition principle, all space-time data can be considered as infinite combinations of spatial patterns and associated time series. Therefore, bandpass filtering over any frequency band would yield modes that vary primarily across the time scale that was isolated. This begs the following question: why is the MJO significant if it appears as though it must be extracted via filtering before it can be observed?

By performing extended EOF analysis on the prefiltered zonal velocity data, it will be clear that the MJO, while not as energetic as the annual cycle of solar radiation or the Indian monsoon mode, is indeed embedded in the data and does account for a nontrivial portion of the variance. A lag covariance parameter of 15 pentads is used, which corresponds to a maximum lag of
75 days. Varying this parameter between 5 and 21 pentads did not materially affect the results. Annual and semiannual variations associated with the seasonal march of solar radiation are not removed. Given its propagating nature, it is anticipated that MJO variability will be represented by two extended EOF modes in temporal quadrature with each other. Cross correlation and hodograph analysis will be utilized to test for quadrature. Spectral and wavelet analyses (see Meyers et al. 1993) will be conducted on the leading PC time series of MJO variability to ascertain the energetic time scales captured by QuikSCAT, as well as how the time scale fluctuates over the record length. In addition, the above analysis will be repeated with the National Centers for Environmental Prediction (NCEP) Reanalysis 2 zonal wind data (see Kanamitsu et al. 2002), provided by the National Oceanic and Atmospheric Administration-Cooperative Institute for Research in Environmental Sciences (NOAA-CIRES) Climate Diagnostics Center directly from their Web site (http://www.cdc. noaa.gov/). This dataset covers the same time period (late July 1999 through early December 2004) and was linearly interpolated and regridded to the $1^{\circ} \times 1^{\circ}$ grid of the QuikSCAT data in order to compare extraction of the MJO using these two independent data sources.

\section{b. Results}

Our results indicate that the primary modes of zonal velocity over our domain are associated with the annual cycle, the transitions between summer and winter Indian monsoons, and semiannual variations. These three phenomena are associated with the three leading modes of prefiltered zonal wind, accounting for $41.5 \%$, $10.1 \%$, and $3.7 \%$ of the total variance, respectively. However, modes 4 and 5, in quadrature with each other, are clearly associated with the MJO. This is made evident by examining the lagged eigenfunctions of the fourth mode (Fig. 1a) as well as the associated PC time series (Fig. 1b). Based on the extended EOF maps, the pattern propagates eastward at approximately $4 \mathrm{~m} \mathrm{~s}^{-1}$. This compares favorably with previous reports (Hendon and Salby 1994; Jones and Weare 1996; Shinoda et al. 1998; Rui and Wang 1990). Together, modes 4 and 5 account for about $4.6 \%$ of the total variance, a respectable portion considering the analysis was performed on the actual zonal velocity. EOF analysis of the nonfiltered zonal velocity data was also performed (not shown). As expected, EOF analysis was not capable of separating the MJO's intraseasonal, eastward-propagating variability from lower-frequency modes.

The spectral characteristics of the PC time series in Fig. $1 \mathrm{~b}$ are shown in Fig. 2. The spectral peak occurs near the 50-day time scale, in general agreement with 
(a)
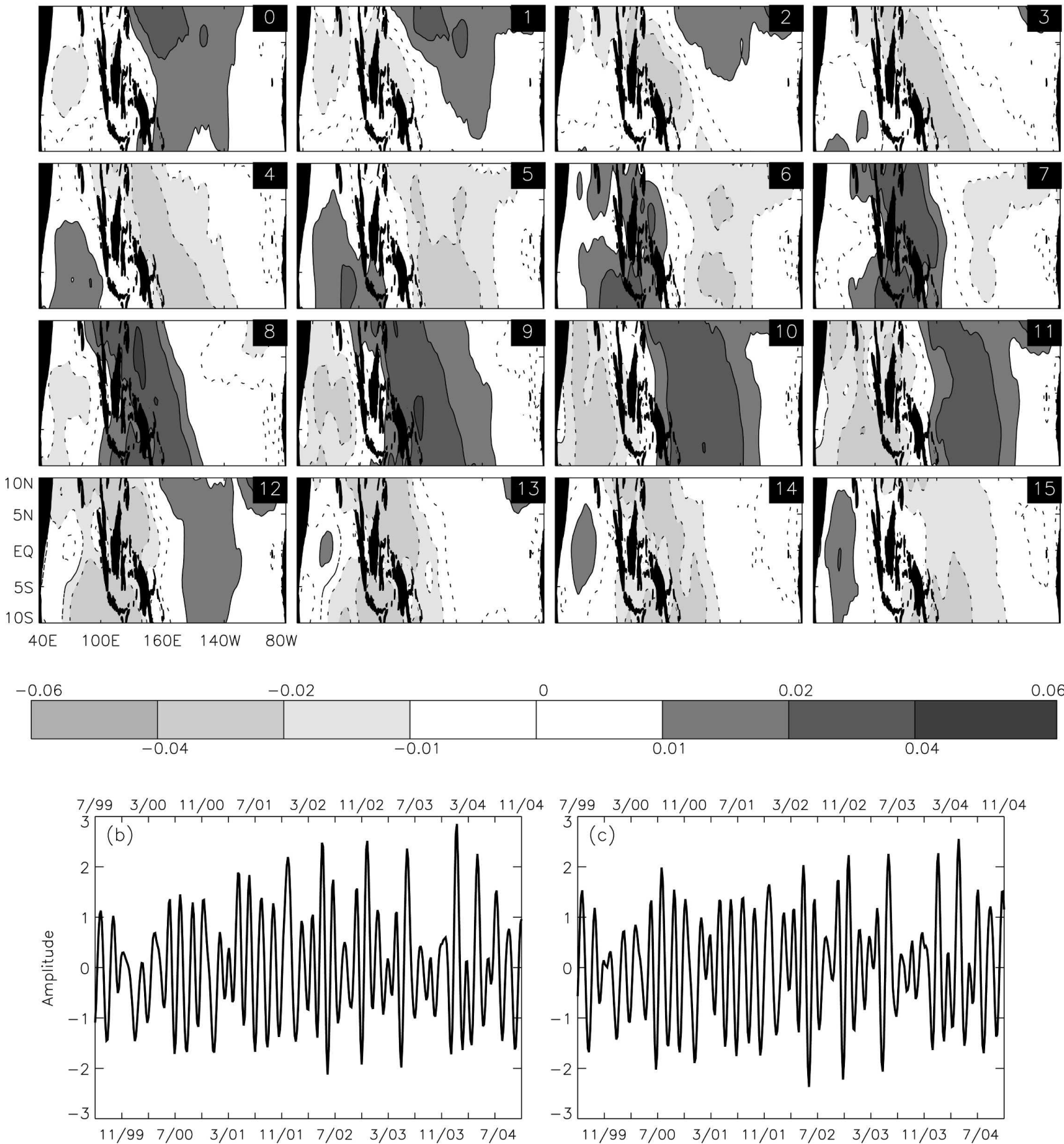

FIG. 1. (a) Lagged eigenfunctions of the fourth leading extended EOF (EXEOF) of nonfiltered zonal velocity from QuikSCAT. The lag parameter for the EXEOF analysis was 15 pentads. Negative contours are dashed and the zero line is bold. Land areas, including the Maritime Continent and Africa in the west, are shown in black. The lag number is shown in the upper right-hand corner of each plot. Note that plots 9-10 lags apart are similar, indicating an MJO time scale of 45-50 days. (b) The associated PC time series. (c) The PC time series of the sixth leading mode of EXEOF analysis using NCEP Reanalysis 2. The time series in (b) and (c) have a maximum lag correlation coefficient of 0.96 . 

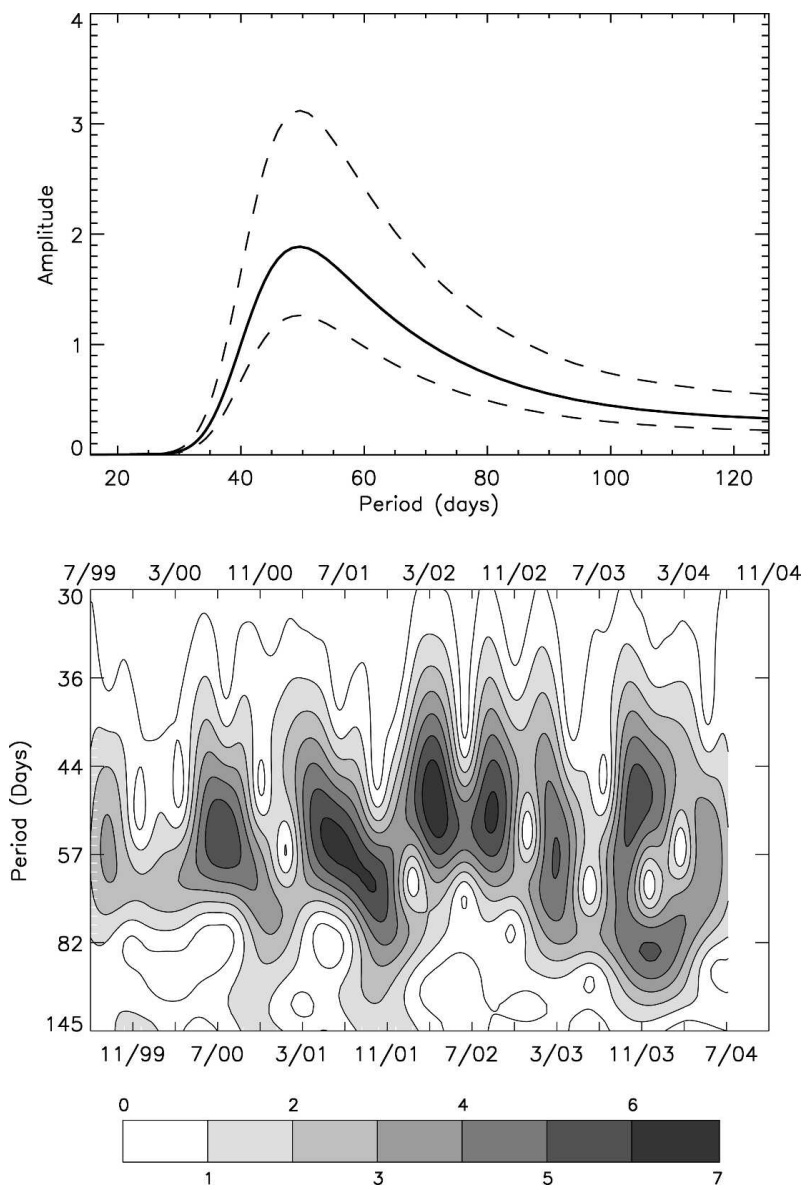

FIG. 2. (a) Smoothed spectral estimates of the time series in Fig. 1b. The $95 \% \chi^{2}$ confidence bands are shown as dashed lines. (b) Wavelet analysis of the time series in Fig. 1b. The values have been scaled to range between 0 and 7 .

previous observations of the MJO time scale (see Madden and Julian 1994). Wavelet analysis provides an idea of the variation in time scale (Fig. 2b). Clearly, the time scale fluctuates around 50 days, with considerable energy occurring between 35 - and 85 -day periods in certain instances.

\section{c. Comparison with NCEP Reanalysis 2}

Extended EOF analysis of the nonfiltered zonal velocity from the National Centers for Environmental Prediction (NCEP) Reanalysis 2 reveals a clear MJO pair as modes 6 and 7 , garnering a combined $3.2 \%$ of the variability. The PC time series of mode 6 is presented in Fig. 1c. Its spectrum and a wavelet analysis of this time series are presented in Fig. 3. The PC time series has a maximum lagged correlation (at 1 pentad) of 0.96 with the fourth leading mode PC from QuikSCAT, shown in Fig. 1b. In addition, the raw and wave-
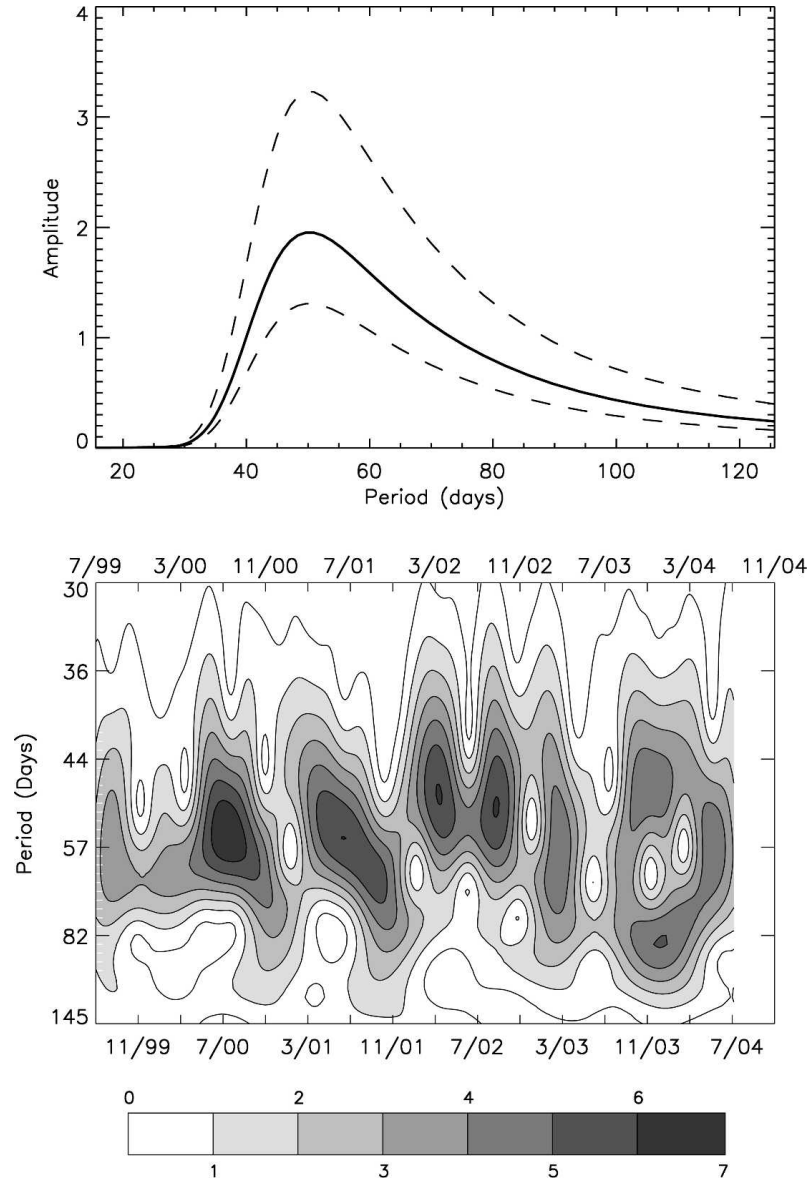

FIG. 3. As in Fig. 2, but for the PC time series of the sixth leading mode of zonal wind using NCEP Reanalysis 2.

let spectra in Fig. 3 for the reanalysis data compare remarkably well with the corresponding QuikSCAT plots in Fig. 2, both showing clear spectral peaks at a time scale of 50 days and evincing common fluctuations in spectral energy over time.

The difference in percent variance accounted for by the MJO modes in QuikSCAT (4.5\%) and NCEP Reanalysis $2(3.2 \%)$ is noteworthy. This represents an increase of relative importance of about $40 \%$ from one dataset to another. If we account for the fact that the QuikSCAT data overall contain a 9\% larger total variance over the MJO domain than the reanalysis data prior to interpolation (20\% greater after interpolation), the disparity in intraseasonal energy between the two datasets is even greater. Spectral energy over the MJO domain in the 30-60-day range is about 30\% lower on average in the Reanalysis zonal wind data compared to QuikSCAT data (Fig. 4). Therefore, it appears that the major difference between observed MJOs in QuikSCAT and NCEP Reanalysis 2 lies in the signals' amplitudes, whereas strong agreement exists regarding the 


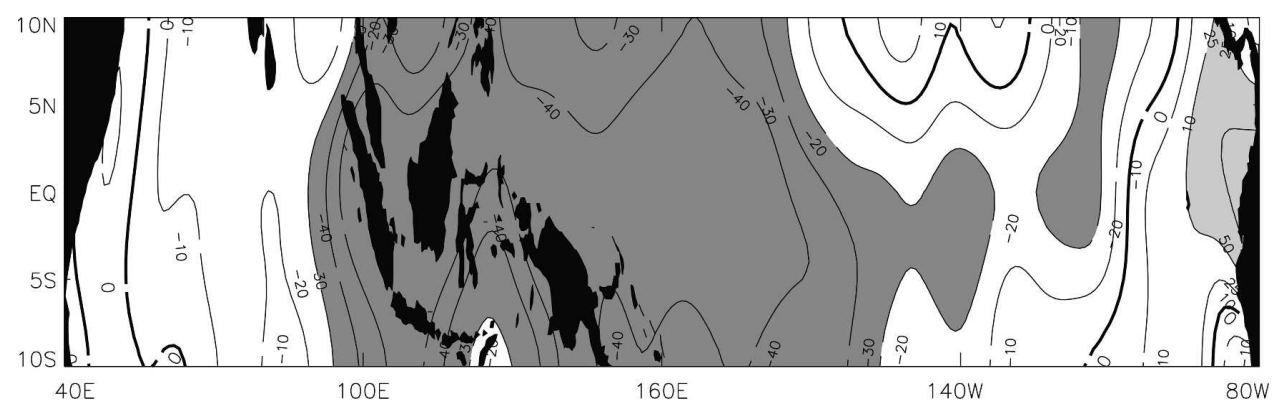

FIG. 4. Local percent deviation of spectral energy in the 30-60-day band for zonal winds between QuikSCAT and NCEP Reanalysis 2. Areas with light shading indicate regions where the spectral energy is at least $25 \%$ greater in the reanalysis than QuikSCAT. Darker shading indicates that QuikSCAT has at least $25 \%$ more spectral energy than the reanalysis. It is clear that QuikSCAT zonal wind data displays considerably larger spectral energy within MJO time scales, especially near the Maritime Continent and in the western Pacific Ocean.

observed time scales as well as temporal fluctuations in time scale.

\section{Bandpass-filtered analyses}

\section{a. Methodology}

Based on the spectrum in Fig. 2a, Lanczos weights were determined for bandpass filtering $u, \chi$, and $\tau^{x}$. The filter consists of 31 weights, symmetric about the central point, with half power at about 33 and 75 days. Timelongitude plots are computed from the bandpassfiltered values of $u, \chi$, and $\tau^{x}$. From these charts, propagation speeds are estimated.

To preserve the record length, which is pivotal when dealing with satellite data because of limited record lengths, a minimization algorithm is employed that determines filter weights for computing values for each slot in the endpoints (see the appendix). Wheeler and Hendon (2004) point out that the major obstacle of real-time monitoring and prediction of the MJO is the loss of endpoints due to bandpass filtering. The variable filter weight technique offered here is an attractive alternative to the more indirect methods currently used to address this issue.

\section{b. Results}

Time-longitude plots of bandpass-filtered $u, \chi$, and $\tau^{x}$ are shown in Fig. 5. Clear eastward propagation is evident for each variable. Approximate estimations from Fig. 5 suggest a propagation speed across the longitudinal extent of our domain of about $5 \mathrm{~m} \mathrm{~s}^{-1}$. However, it is clear that the propagation speed is not at a constant rate but ranges from about 1 to $10 \mathrm{~m} \mathrm{~s}^{-1}$. This compares favorably with previous studies (e.g., Lau and Chan 1985). Closer inspection reveals that the propagation speed is considerably higher in the eastern Pacific $(\sim 10$ $\mathrm{m} \mathrm{s}^{-1}$ ), in accordance with previous research (Hendon and Salby 1994; Maloney and Hartmann 1998).

The time-longitude plots portray fairly regular MJO events, although the MJO signal was either absent or diminished during several brief episodes. These include disruptions in early 2000 and late 2003, when a westward propagation occurred, as well as a brief absence in late summer of 2002. In general, it appears that the MJO is not prone to favor a particular season, although recent studies maintain that the signal is most conspicuous during springtime because of reduced interaction with the Indian monsoon (e.g., Myers and Waliser 2003). Similar time-longitude analyses were conducted with other kinematic variables, such as meridional velocity, and rotational quantities, such as vorticity and streamfunction. As expected from previous works, clear MJO signals were not apparent in these parameters.

\section{Summary and conclusions}

Gridded data from the SeaWinds instrument on the QuikSCAT satellite were inspected to ascertain how well the instrument could resolve MJO variability at the surface. Extended EOF analysis of nonfiltered surface zonal velocity over the tropical Indian and Pacific Oceans, with a lag parameter of 15 pentads, reveals that MJO variability is captured by modes 4 and 5 . These two modes are in quadrature with each other and explain a combined $4.6 \%$ of the total variance. The first three modes explain the majority of the variance and are associated with annual, semiannual, and monsoonrelated variations. Although the MJO is not the dominant mode in the nonfiltered dataset, it does represent the dominant mode of intraseasonal variability, overshadowed by modes associated primarily with the annual cycle of solar radiation. The QuikSCAT results 
(a)

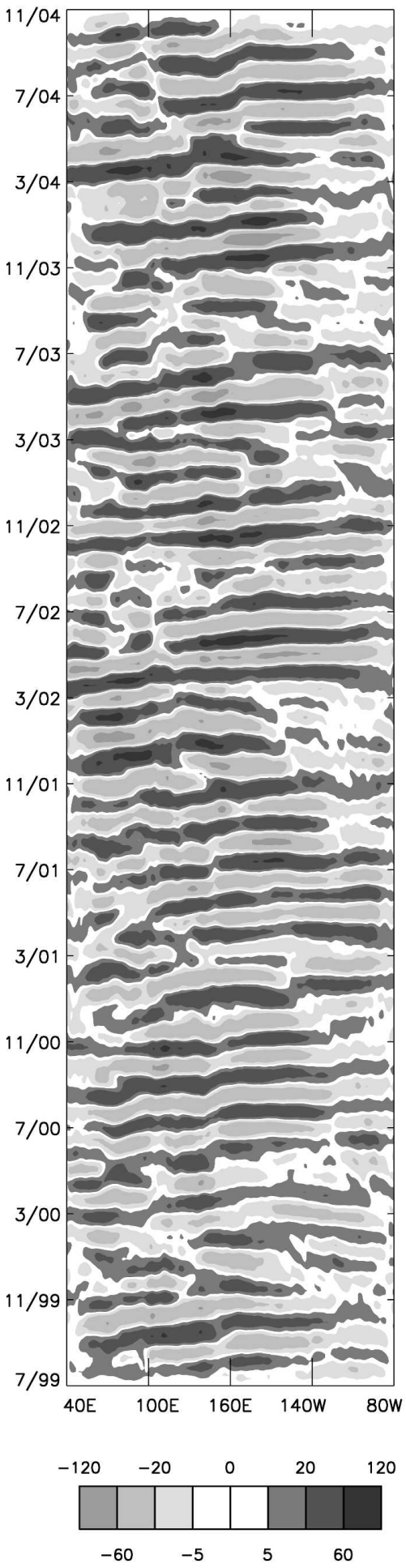

(b)

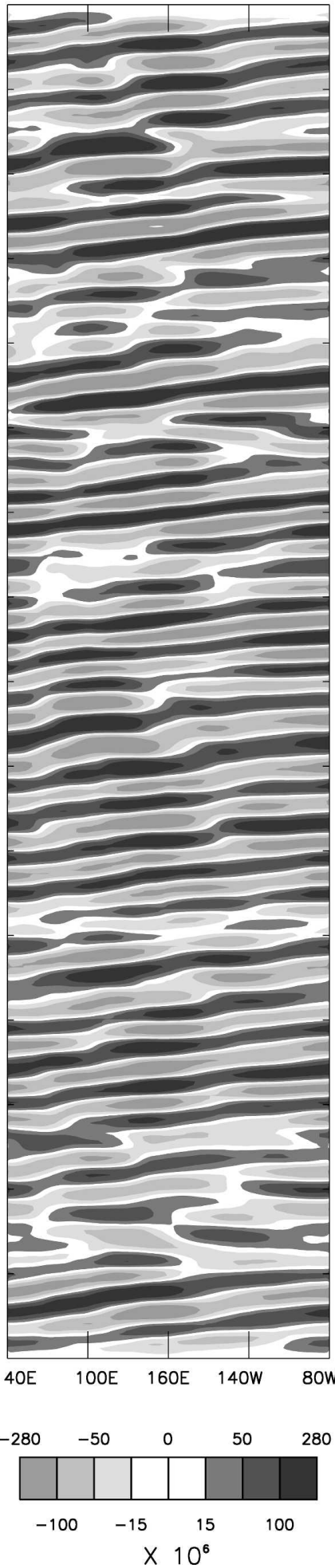

(c)

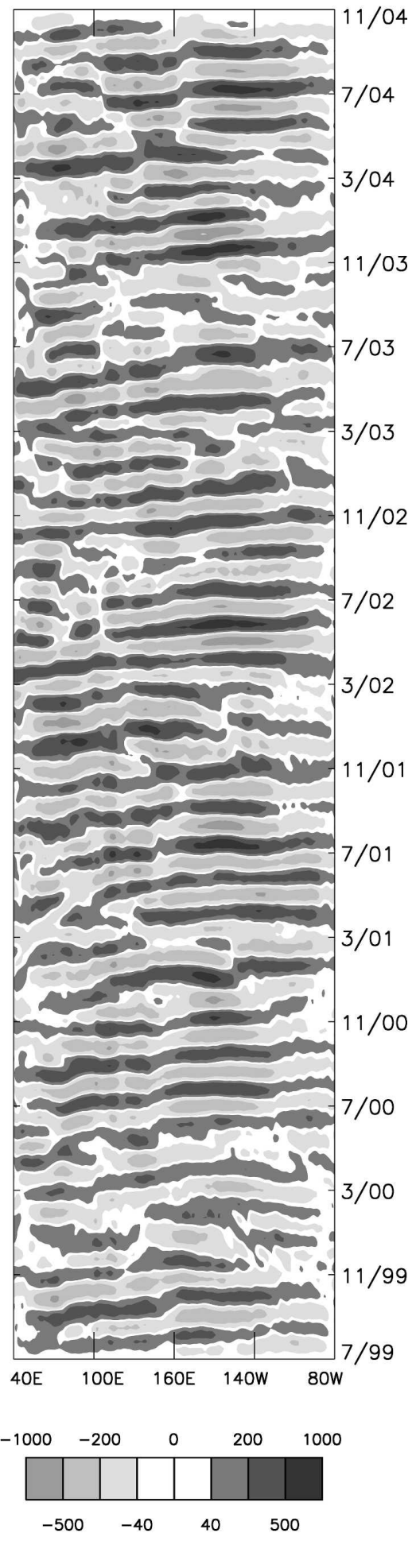

FIG. 5. (a) Hovmöller diagram of bandpass-filtered $u$. (b) Same as in (a), but for velocity potential. (c) Same as in (a), but for zonal pseudostress. For all three charts, the magnitudes are arbitrary and dimensionless. This is a consequence of bandpass filtering with the constraint of preserving the expectation of the mean of the data. 
compare favorably with results from NCEP Reanalysis 2 , with the main difference being the considerably larger amplitude of the MJO's surface signal in QuikSCAT data.

After confirming the existence of MJO variability in the data, bandpass filtering was conducted to isolate MJO time scales. Clear MJO patterns (with appropriate spatial scales and eastward propagations) were found in zonal velocity, velocity potential, and zonal pseudostress. Time-longitude plots and extended EOF analysis reveals typical propagation speeds ranging from 1 to $10 \mathrm{~m} \mathrm{~s}^{-1}$, with a mean value in the vicinity of $4 \mathrm{~m} \mathrm{~s}^{-1}$.

In addition, an alternative filtering strategy was applied that retained time series endpoints, based on least squares minimization of the frequency response function. This generalized filtering method is not unique to bandpass filters, such as the Lanczos filters, since the frequency response function is a fundamental attribute of all filters. We anticipate that this method can be utilized in other climate research avenues, especially for shorter record lengths like satellite datasets. With regard to the $\mathrm{MJO}$, it is anticipated that real-time monitoring and perhaps even prediction of the MJO can benefit from this method.

Our findings highlight the usefulness of a gridded QuikSCAT dataset for investigation of the MJO. As the steady stream of satellite data, such as those available from QuikSCAT, further improve the spatial and temporal coverage of available datasets, more detailed descriptions of weather phenomena will be possible. In particular, the potential of improved understanding of the MJO due to increased coverage, rather than relying on reanalysis data, is promising, especially for winds.

Acknowledgments. Primary funding for this work was obtained from the Center for Ocean-Atmospheric Prediction Studies, which receives its base funding from NOAA's Office of Global Programs. The Florida State University Office of Graduate Studies, the National Science Foundation, the NASA/OSU SeaWinds project, Remote Sensing Systems, and NASA Earth Science Enterprise provided additional support. Additional thanks go to Peng Yu, Kwang-Yul Kim, Jorge Zavala-Hidalgo, Shawn Smith, Ajaya Mohan-Ravin- dran, Yan $\mathrm{Gu}$, and several anonymous reviewers for their valuable advice and support.

\section{APPENDIX}

\section{Time Series Filtering near Endpoints}

Time series filtering is typically computed in time space, using filter weights, $h(\tau)$. This requires a convolution,

$$
y(t)=\sum_{\tau=a}^{\tau=b} h(\tau) x(t+\tau) .
$$

The parameters $a$ and $b$ are integers that are usually chosen such that $b$ is the negative of $a$. Thus, the number of filter weights becomes $2 b+1$. The parameter $\tau$ represents a time lag. Filtering in the time domain results in lost points at the endpoint intervals; this is a consequence of using a convolution. Specifically, the convolution cannot be defined at the first $b$ points and the last $b$ points of the time series. In these regions, at least one of the lags is associated with an unavailable point in the time series (points beyond the terminal values). These points where the full convolution cannot be computed are usually dropped from consideration (see Fig. A1a).

In the present study, variable filter weights in the endpoint intervals are determined such that the frequency response function (FRF) appropriately reproduces the predetermined FRF of the interior points. A penalty function is constructed that minimizes the squared error between the FRF in the interior and a new FRF in each point within the endpoint intervals. The value of $b$ is incrementally decreased to force a symmetric filter at each point. The result is a set of filter weights for each point in the endpoint interval. It can be shown that, unconstrained, this minimization technique will simply truncate the filter weights used in the interior (see Bloomfield 2000). A practical constraint is to force the new weights to sum to 1 , thereby preserving the expectation of the mean of the output series. For a particular value of $b, L$, referring to a point in the endpoint interval, the new FRF is

$$
G(f)=\sum_{k=-L}^{L} \alpha(k) e^{i 2 \pi f k}
$$

FIG. A1. Schematic used for describing the variable filter weight technique. (a) Sample 100-point time series to be filtered with a 31-point Lanczos filter. Points marked with an asterisk depict time points in which the complete convolution can be computed with 31 weights. In the left- and right-hand edges of the time series (the first and last 15 points of the time series), the least squares filtering technique is employed. (b) The full 31 Lanczos weights used for bandpass filtering in the text. (c)-(e) Filter weights computed using the least squares technique at points 11, 7, and 3 in the time series, respectively. The same weights are used at the corresponding points in the right-hand end of the series. (f)-(i) The associated power transfer functions of the filter weights in the left-hand panel. 

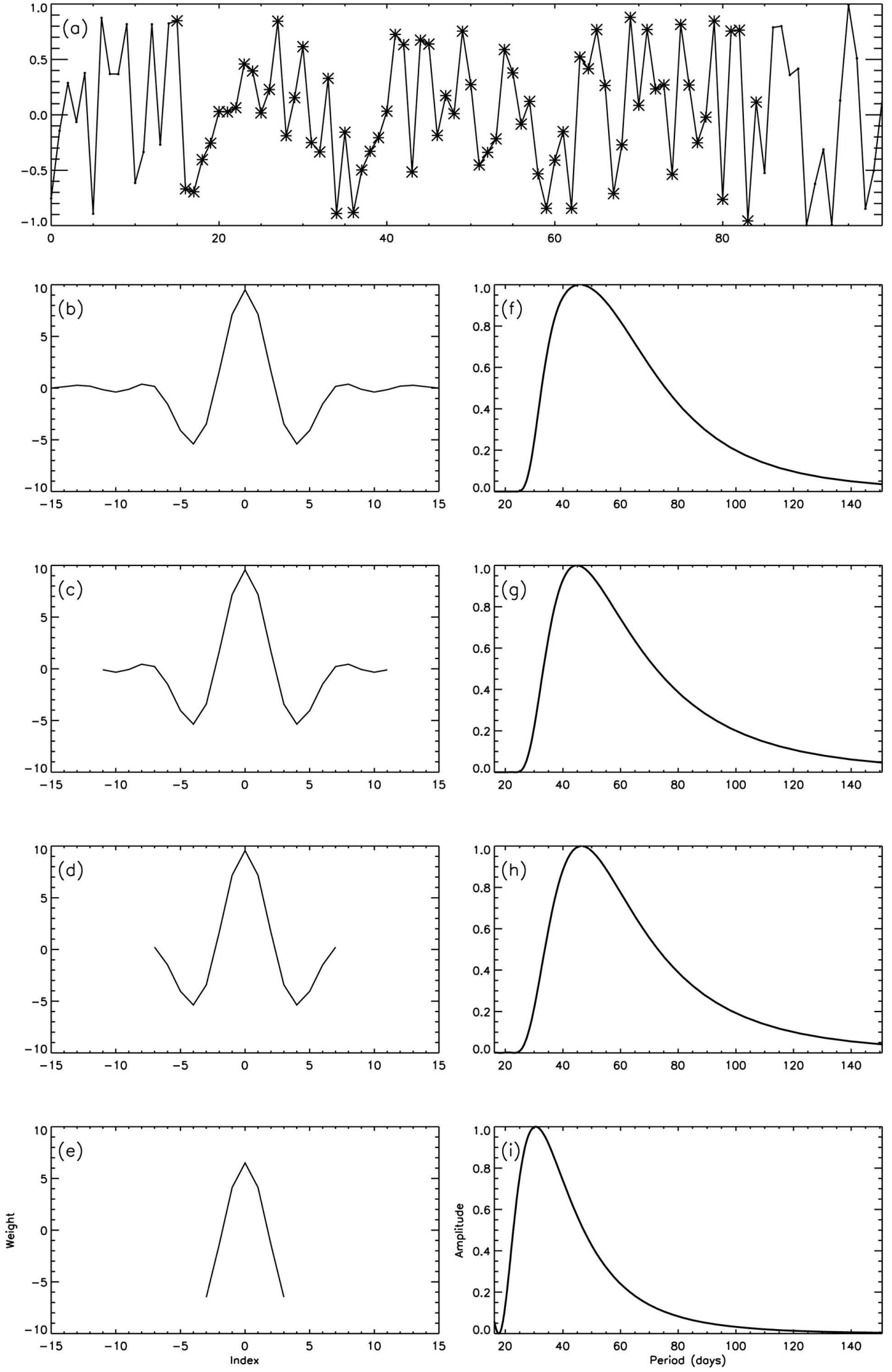

FIg. A1. (Continued) 
where $\boldsymbol{\alpha}$ represents the new filter weights. The cost function $J$ for a given point $L$ in the endpoint interval is a function of $\boldsymbol{\alpha}$ and $\lambda$ (the Lagrangian multiplier that imposes our constraint):

$$
J(\boldsymbol{\alpha}, \lambda)=\sum_{f}[H(f)-G(f)]^{2}+\lambda\left[1-\sum_{k=-L}^{L} \boldsymbol{\alpha}(k)\right],
$$

where $H$ represents the frequency response function of interior points. The minimum of this penalty function is obtained by taking the partial derivatives with respect to each $\alpha$ and $\lambda$ and setting them equal to zero:

$$
\begin{aligned}
& \frac{\partial J}{\partial \alpha_{j}}=-2 \sum_{f}[H(f)-G(f)] e^{i 2 \pi f j}-\lambda=0, \\
& \frac{\partial J}{\partial \lambda}=1-\sum_{k=-L}^{L} \alpha(k)=0 .
\end{aligned}
$$

Equations (A4) and (A5) are solved simultaneously using matrix operations to arrive at the coefficients.

Figure A1 shows the filter weights for the complete 31-point Lanczos filter (Fig. A1b), as well as the computed weights for points 11,7 , and 3 in the time series (Figs. A1c-e, respectively). Figures A1f-i show the associated power transfer functions. Inevitably, as indicated in the figure, the errors due to this method increase closer to the terminal points for two primary reasons. First, less data are available for the computation. Second, the ability to reproduce the frequency response function is hampered by a reduced number of allowed filter weights. The second source of error is especially problematic for bandpass filters, such as the Lanczos filter used in the present study, since the weights must include both positive and negative weights to extract salient time scales. Despite these errors, it is the authors' contention that the method presented here for estimating endpoint values is preferable to discarding endpoints, a common practice in the geosciences. Future work may involve combining the least squares technique with a statistical prediction method that takes on increasing weight closer to the terminal ends.

\section{REFERENCES}

Bloomfield, P., 2000: Fourier Analysis of Time Series-An Introduction. John Wiley and Sons, $261 \mathrm{pp}$.

Bourassa, M. A., D. M. Legler, J. J. O'Brien, and S. R. Smith, 2003: SeaWinds validation with research vessels. J. Geophys. Res., 108, 1-16.

Hendon, H. H., and M. L. Salby, 1994: The life cycle of the Madden-Julian oscillation. J. Atmos. Sci., 51, 2225-2237.

Jones, C., and B. C. Weare, 1996: The role of low-level moisture convergence and ocean latent heat fluxes in the Madden and Julian oscillation: An observational analysis using ISCCB data and ECMWF analyses. J. Climate, 9, 3086-3104.
Kanamitsu, M., W. Ebisuzaki, J. Woollen, S. K. Yang, J. J. Hnilo, M. Fiorino, and G. L. Potter, 2002: NCEP-DOE AMIP-II Reanalysis (R-2). Bull. Amer. Meteor. Soc., 83, 1631-1643.

Kayano, M. T., and V. E. Kousky, 1998: Zonally symmetric and asymmetric features of the tropospheric Madden-Julian oscillation. J. Geophys. Res., 103, 13 703-13 712.

Kessler, W. S., 2001: EOF representation of the Madden-Julian oscillation and its connection with ENSO. J. Climate, 14, 3055-3061.

Krishnamurti, T. N., D. K. Oosterhof, and A. V. Mehta, 1988: Air-sea interaction on the time scale of 30 to 50 days. $J$. Atmos. Sci., 45, 1304-1322.

-, H. S. Bedi, and V. M. Hardiker, 1998: An Introduction to Global Spectral Modeling. Oxford University Press, 253 pp.

Lau, K.-M., and P. H. Chan, 1985: Aspects of the 40-50 day oscillation during the northern winter as inferred from outgoing longwave radiation. Mon. Wea. Rev., 113, 1889-1909.

Madden, R. A., and P. R. Julian, 1971: Description of a 40-50 day oscillation in the zonal wind in the tropical Pacific. J. Atmos. Sci., 28, 702-708.

—, and - 1994: Observations of the 40-50-day tropical oscillation-A review. Mon. Wea. Rev., 122, 814-837.

Maloney, E. D., and D. L. Hartmann, 1998: Frictional moisture convergence in a composite life cycle of the Madden-Julian oscillation. J. Climate, 11, 2387-2403.

Meyers, S. D., B. G. Kelly, and J. J. O'Brien, 1993: An introduction to wavelet analysis in oceanography and meteorology: With application to the dispersion of Yanai waves. Mon. Wea. Rev., 121, 2858-2866.

Myers, D. S., and D. E. Waliser, 2003: Three-dimensional water vapor and cloud variations associated with the MaddenJulian oscillation during Northern Hemisphere winter. J. Climate, 16, 929-950.

Pegion, P. J., M. A. Bourassa, D. M. Legler, and J. J. O'Brien, 2000: Objectively derived daily "winds" from satellite scatterometer data. Mon. Wea. Rev., 128, 3150-3168.

Raymond, D. J., 2001: A new model of the Madden-Julian oscillation. J. Atmos. Sci., 58, 2807-2819.

Rui, H., and B. Wang, 1990: Development characteristics and dynamic structure of tropical intraseasonal convection anomalies. J. Atmos. Sci., 47, 357-379.

Schlax, M. G., D. B. Chelton, and M. H. Freilich, 2001: Sampling errors in wind fields constructed from single and tandem scatterometer datasets. J. Atmos. Oceanic Technol., 18, 10141036.

Shinoda, T., H. H. Hendon, and J. Glick, 1998: Intraseasonal variability of surface fluxes and sea surface temperature in the tropical western Pacific and Indian Oceans. J. Climate, 11, $1685-1702$

Weare, B. C., and J. S. Nasstrom, 1982: Examples of extended empirical orthogonal function analyses. Mon. Wea. Rev., 110, 481-485.

Weissman, D. E., M. A. Bourassa, and J. Tongue, 2002: Effects of rain rate and wind magnitude on SeaWinds scatterometer wind speed errors. J. Atmos. Oceanic Technol., 19, 738-746.

Wentz, F. J., and D. K. Smith, 1999: A model function for the ocean-normalized radar cross section at $14 \mathrm{GHz}$ derived from NSCAT. J. Geophys. Res., 104, 11 499-11 514.

Wheeler, M. C., and H. H. Hendon, 2004: An all-season real-time multivariate MJO index: Development of an index for monitoring and prediction. Mon. Wea. Rev., 132, 1917-1932. 